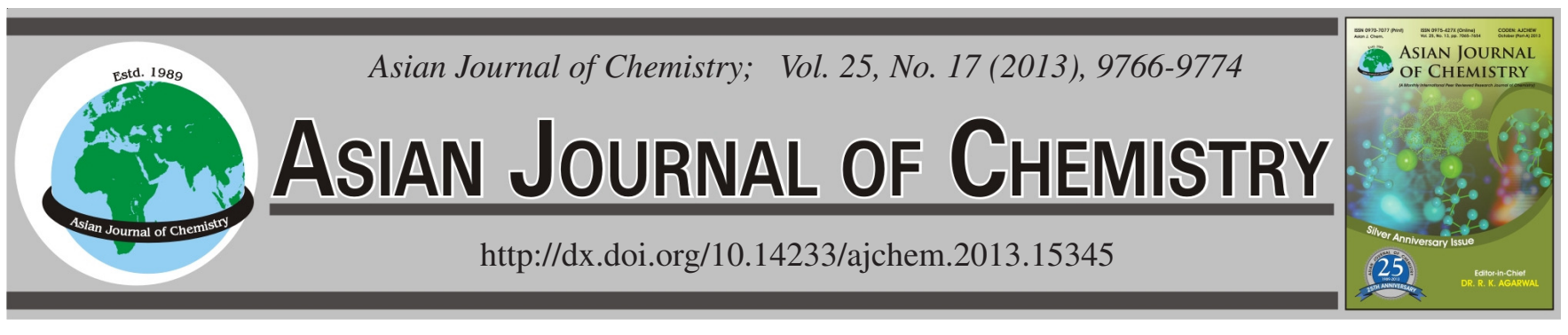

\title{
Synthesis and Characterization of Titanium Dioxide Nano Particles and their Application in Environmental Remediation of Textile Dyes
}

\author{
Naima Termazi ${ }^{1}$, Farhat Abbas ${ }^{1, *}$, Iftikhar Hussain Bukhari ${ }^{2, *}$, Tariq Mahmood Bhatti ${ }^{3}$, Muhammad Riaz $^{2}$, \\ Muhammad Aftab Rafiq ${ }^{4}$, Qaiser M. Khan ${ }^{5}$, Mnaza Noreen ${ }^{2}$ and Yasmeen Gull ${ }^{2}$
}

\begin{abstract}
${ }^{1}$ Department of Environmental Sciences, Government College University, Faisalabad-38000, Pakistan
${ }^{2}$ Department of Chemistry, Government College University, Faisalabad-38000, Pakistan

${ }^{3}$ Department of Chemical Engineering, Pakistan Institute of Engineering and Applied Sciences, PIEAS Nilore, Islamabad, Pakistan

${ }^{4}$ Department of Materials and Metallurgical Engineering, Pakistan Institute of Engineering and Applied Sciences, PIEAS Nilore, Islamabad, Pakistan ${ }^{5}$ Environmental Biotechnology Division, National Institute for Biotechnology and Genetic Engineering, P.O. Box 577, Jhang Road, Faisalabad, Pakistan
\end{abstract}

*Corresponding author: E-mail: farhat@gcuf.edu.pk; pdiftikhar@yahoo.com

\begin{abstract}
The waste water of different textile industries when enter to a water body it impairs that water and inhibits sunlight to reach at the bottom thus affecting flora and also due to process of bioaccumulation and biomagnifications, it can harm fauna and ultimately humans. Moreover this waste water comes into contact with people when this polluted water is used for washing of different edibles as fruits and vegetables. In this study we synthesized titanium dioxide nanoparticles through co-precipitation method and tested their effect on remediation of different dyes. For this purpose we took reactive azo dyes from a manufacturing company and make synthetic effluents. Samples were prepared in accordance to the standard procedures and protocols.
\end{abstract}

Key Words: Synthesis, Titanium dioxide, Nano particles, Remediation, Textile dyes.

\section{INTRODUCTION}

Nanoparticles can be used in industry, technology, medicine, agriculture, engineering, environment and many others, their uses are limited only due to human imagination. Textile is basic necessity of life that's why textile industry constitutes a great proportion of industrial sector. Large amounts of water are used for dyeing and finishing of fabrics in the textile industries. $1.08-0.15 \mathrm{~m}^{3}$ of clean water is consumed in producing 1 kilogram of finished cloth material, 1,000-3000 $\mathrm{m}^{3}$ wastewater generates each day for production of 12-20 ton of adjourned fabric per day ${ }^{1}$.

Water is a basic necessity for life itself. A great number of chemicals and organic substances are introduced in water from sources as chemical spills, agricultural runoff and industrial effluents ${ }^{2}$. The stability of these chemicals to natural decomposition, their toxicity and their persistence in environment is most concerning issue to the societies and regulation authorities round the globe.

Removal of colour from textile mills polluted water has become an alarming issue for the duration of last few decades due to the dyes which are used in textile industries to colour the fabric not only are toxic but also impair the visibility of receiving waters. Great values of BOD and COD, the presence of particulate matter, sediments and dark coloured chemicals make effluents turbid. Due to these actions depletion of dissolved oxygen (DO) occurs which put adverse impacts on marine ecological system. Dyes are unchangeable in chemical and photolytic nature and are exceedingly obstinate in natural environs. These chemicals can cause different allergies of skin as irritation, chemical injuries, ulcers and problems of respiration $^{3,4}$.

Nearly $10-15 \%$ of dyes are lost in the dyeing processes ${ }^{5}$ and are released in the wastewater causing environmental contamination $^{6}$. The azo dyes are mutagenic and carcinogenic in nature and have recalcitrant properties. In last two decades titania nanoparticles have been proved useful for wastewater remediation through photocatalysis as other conventional methods as biological methods are no longer useful due to the change in properties of dyes now out of 87 dyes $53 \%$ are non biodegradable $\mathrm{e}^{7,8}$.

Bui and coworkers showed that the photo catalysis potential through using $\mathrm{TiO}_{2}$ powder for environmental remediation of two azo dye and results showed the complete mineralization of Reactive Red 4 and Reactive Black $5^{9}$. The $\mathrm{TiO}_{2}$ performance as a photocatalytic degrader ${ }^{10}$. It observed 
that nano particles of $\mathrm{TiO}_{2}$ in the presence of UV for the degradation of methylene blue in two batch slurry reactors ${ }^{11}$.

\section{EXPERIMENTAL}

Synthesis: Titanium dioxide nanoparticles were synthesized through physical method of co-precipitation. We took $21 \mathrm{~mL}$ of titanium tetrachloride which was $98 \%$ pure. $51 \mathrm{~mL}$ of 0.5 $\mathrm{M}$ hydrochloric acid was taken in beaker and was stirred. $\mathrm{TiCl}_{4}$ was added drop wise in it. This newly prepared solution was stirred for some time. 2.1 M solution of sodium carbonate was prepared to neutralize the $\mathrm{TiCl}_{4}$. Now the $\mathrm{pH}$ of $\mathrm{TiCl}_{4}$ and $\mathrm{HCl}$ solution was neutralized by adding $\mathrm{Na}_{2} \mathrm{CO}_{3}$ in it dropwise until the $\mathrm{pH}$ reached at 8.5. At $\mathrm{pH} 8.5$ precipitates of $\mathrm{TiO}_{2}$ formed so addition of $\mathrm{Na}_{2} \mathrm{CO}_{3}$ was stopped.

These precipitates were washed with distilled water to remove $\mathrm{Na}^{+}$ions. After $2 \mathrm{~h}$ of washing precipitates were centrifuged which were then dried at $110^{\circ} \mathrm{C}$ for $24 \mathrm{~h}$. After drying particles were calcined at $200{ }^{\circ} \mathrm{C}$ for $2 \mathrm{~h}$ to achieve anatase phase. After calcination particles were grinded to make powder.

Precautions: $\mathrm{TiCl}_{4}$ is toxic and can react with moisture so the taking of it was done in glove box in low humidity. More the precipitates are washed, more the impurities are removed. Precipitates were centrifuged to separate water from it. Avoid contact of $\mathrm{TiCl}_{4}$ with water as it can explode.

Characterization of titania nanoparticles by XRD: The XRD pattern of $\mathrm{TiO}_{2}$ was obtained using X-ray diffractometer: Brucker DB with $\mathrm{CuK}_{\alpha}$ radiation in the range of $20-70^{\circ}(\lambda=$ $0.154 \mathrm{~nm})$.

Treatment: Reactive azo dyes are widely used in textile industry due to their sharp colour and good finish. Four reactive azo dyes were taken from local manufacturing company for treatment named Reactive Orange 13, Reactive Red 190, Reactive Black 5 and Reactive Yellow 42.

Dark treatment: Took eight vials and labeled them. Put $10 \mathrm{~mL}$ of each dye solution in 2 vials, 1 for treatment and another 1 for control. For treatment $0.05 \mathrm{~g}$ of titania nano particles were added to each dye vial and capped it. These vials were put in shaker for shaking for $10 \mathrm{~min}$ so that nanoparticles mix evenly in solution. Covered these tubes with aluminium foil to prevent exposure to light. Now put these eight test tubes in dark place for $3 \mathrm{~h}$. These tubes were shaked after continuous time interval for even mixing. After $3 \mathrm{~h}$, these samples were centrifuged at $10000 \mathrm{rpm}$ for $10 \mathrm{~min}$. Then wavelength was scanned and observed absorbance of treated blank and other samples at UV spectrophotometer. $\mathrm{pH}$ of the samples was also observed.

UV-visible-spectroscopy: Sample was taken in quartz container and its absorbance was taken against distilled water as blank. The absorbance was taken from $200-800 \mathrm{~nm}$ region.

Total organic carbons (TOC): It was determined according to described method ${ }^{12}$.

Standard solution of glucose: To prepare $1000 \mathrm{ppm}$ glucose solution, $1 \mathrm{~g}$ of glucose dissolved in distilled water and make total volume $1 \mathrm{~L}$. For making $250 \mathrm{ppm}, 25 \mathrm{~mL}$ of above $1000 \mathrm{~mL}$ solution is taken and dissolved in $100 \mathrm{~mL}$ of distilled water.

Procedure: $4 \mathrm{~mL}$ each of distilled water (blank), standard solution and samples were taken in TOC vials in triplicate.
$1 \mathrm{~mL}$ of $2 \mathrm{~N} \mathrm{~K}_{2} \mathrm{Cr}_{2} \mathrm{O}_{7}$ and $1.6 \mathrm{~mL}$ of concentrated $\mathrm{H}_{2} \mathrm{SO}_{4}$ were added. Vials were tightly caped, vortexed for $90 \mathrm{~min}$. Then placed them in digester block at $110^{\circ} \mathrm{C}$. These vials were then allowed to cool to room temperature and make volume $10 \mathrm{~mL}$ with distilled water. Vials were tightly caped and again vortex the tubes. The colour intensity was measured at $590 \mathrm{~nm}$ using UV-visible spectrophotometer. By comparing the optical density (OD) of standard with the test samples, the TOC of test samples was calculated.

Chemical oxygen demand (COD): Chemical oxygen demand is the amount of oxygen required to oxidize all organic compounds in effluent. The degree of oxidation depends upon the type of substance, $\mathrm{pH}$ value, temperature reaction time and conc. of oxidizing agent. COD was determined according to described method ${ }^{12}$.

Digestion solution: $10.2 \mathrm{~g}$ of $\mathrm{K}_{2} \mathrm{Cr}_{2} \mathrm{O}_{7}$ (98\% purity), primary standard grade, previously dried at $150{ }^{\circ} \mathrm{C}$ for $2 \mathrm{~h}$ was added to $c a .500 \mathrm{~mL}$ distilled water in a volumetric flask, then $167 \mathrm{~mL}$ conc. $\mathrm{H}_{2} \mathrm{SO}_{4}$ (95-97\% purity) and $33.3 \mathrm{~g} \mathrm{HgSO}_{4}$ (98.5\% purity) were added in it. When dissolved, cooled to room temperature and diluted to $1 \mathrm{~L}$.

Catalyst solution: $25 \mathrm{~g} \mathrm{Ag}_{2} \mathrm{SO}_{4}$ (98.5\% purity) was added in $2.5 \mathrm{~L}$ of conc. $\mathrm{H}_{2} \mathrm{SO}_{4}(95-97 \%$ purity). It was allowed to stand 1 to 2 days to dissolve thoroughly.

\section{Standard solution of potassium hydrogen phthalate}

Standard solution A: The potassium hydrogen phthalate (99.8\% purity) was lightly crushed and then dried to constant weight at $110^{\circ} \mathrm{C} .850 \mathrm{~g}$ of standard dried potassium hydrogen phthalate was dissolved in $500 \mathrm{~mL}$ of distilled water and diluted to $1 \mathrm{~L}$. The COD of the standard solution is $1000 \mathrm{mg} / \mathrm{L}$. A standard curve of diluted solutions containing 100, 200, 300, $400,500,600,700,800,900$ and $1000 \mathrm{mg} / \mathrm{L}$ COD was prepared and was found linear up to $600 \mathrm{mg} / \mathrm{L} \mathrm{COD}$.

Standard solution B: $200 \mathrm{~mL}$ of standard solution A was diluted to $1000 \mathrm{~mL}$ with distilled water (COD $200 \mathrm{mg} / \mathrm{L}$ ). This solution was stable for 4-5 days when refrigerated, but not indefinitely. Thus a weekly preparation was usually done. This standard solution was used with each set.

Procedure: $2 \mathrm{~mL}$ each of distilled water (blank) standard solution $\mathrm{B}$ and samples were taken in COD vials in triplicate. $1.2 \mathrm{~mL}$ of digestion solution and $2.8 \mathrm{~mL}$ of catalyst solution were added. Vials were tightly caped, vortexed and put in COD digester block at $150{ }^{\circ} \mathrm{C}$ for $2 \mathrm{~h}$. These vials were then allowed to cool to room temperature and the colour intensity was measured at $600 \mathrm{~nm}$ using UV-visible spectrophotometer. By comparing the optical density (OD) of standard with the test samples, the COD of test samples was calculated. $\mathrm{pH}$ samples were measured at the sampling time with bench top $\mathrm{pH}$ meter (Accumet, model 25, Denver Instrument Company USA).

\section{RESULTS AND DISCUSSION}

$\mathrm{XRD}$ results show that nanoparticles are of anatase phase and their size ranges from 50 to $70 \mathrm{~nm}$ (Fig. 1).

Fig. 2 depicts the absorbance of Reactive Orange 13 where OD S1 shows the absorbance of original dye without any treatment, OD TDB1 shows the absorbance of dye treated in dark without nanoparticles and OD TDS1 shows the absorbance of 


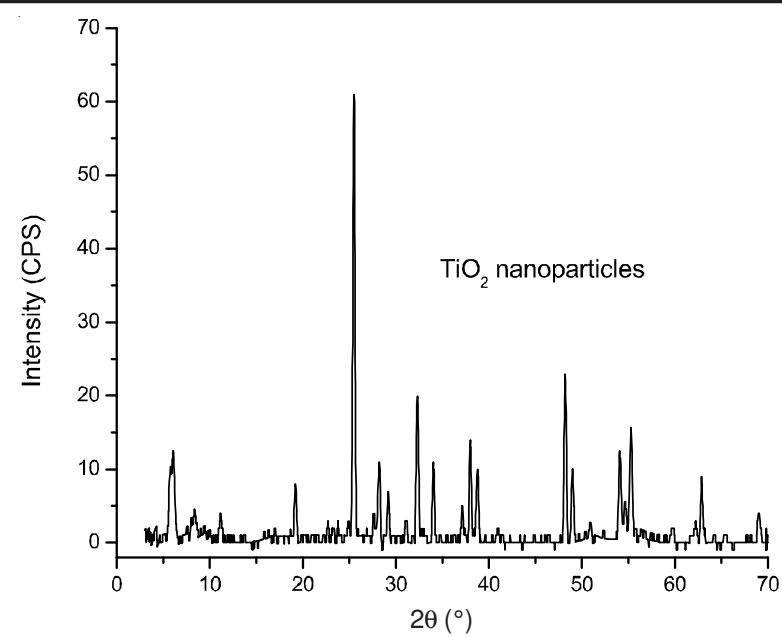

Fig. 1. XRD of titanium dioxide nanoparticles

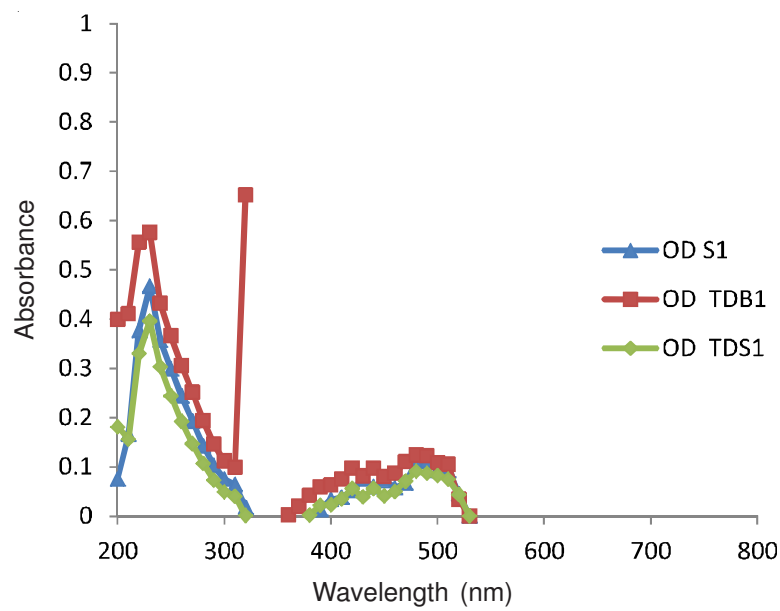

Fig. 2. Nanoparticles in dark changing absorbance of Reactive Orange 13

dye treated in dark in the presence of nanoparticles. The absorbance of dye is maximum when is treated in dark without nanoparticles. It decreases when nanoparticles are added. The effect of nanoparticles on Orange P3R revealed that the absorbance of dye decreased when put in dark for degradation. The absorbance decreased on same trend and no new peaks were formed.

Fig. 3 depicts the absorbance of Reactive Red 190 where OD S1 shows the absorbance of original dye without any treatment, OD TDB1 shows the absorbance of dye treated in dark without nanoparticles and OD TDS1 shows the absorbance of dye treated in dark in the presence of nanoparticles.

The effect of nanoparticles on dye revealed that absorbance of dye decreased when put in dark for degradation. The absorbance decreased on same trend and no new peaks were formed. The treatment decolourized the dye and it took a slight blue shift.

Fig. 4 depicts the absorbance of Reactive Black 5 where OD $\mathrm{S} 1$ shows the absorbance of original dye without any treatment OD TDB1 shows the absorbance of dye treated in dark without nanoparticles and OD TDS1 shows the absorbance of dye treated in dark in the presence of nanoparticles. The effect of nanoparticles on dye revealed that absorbance of dye decreased when put in dark for degradation. The colour of treated dye was faded and there were some changes in it.

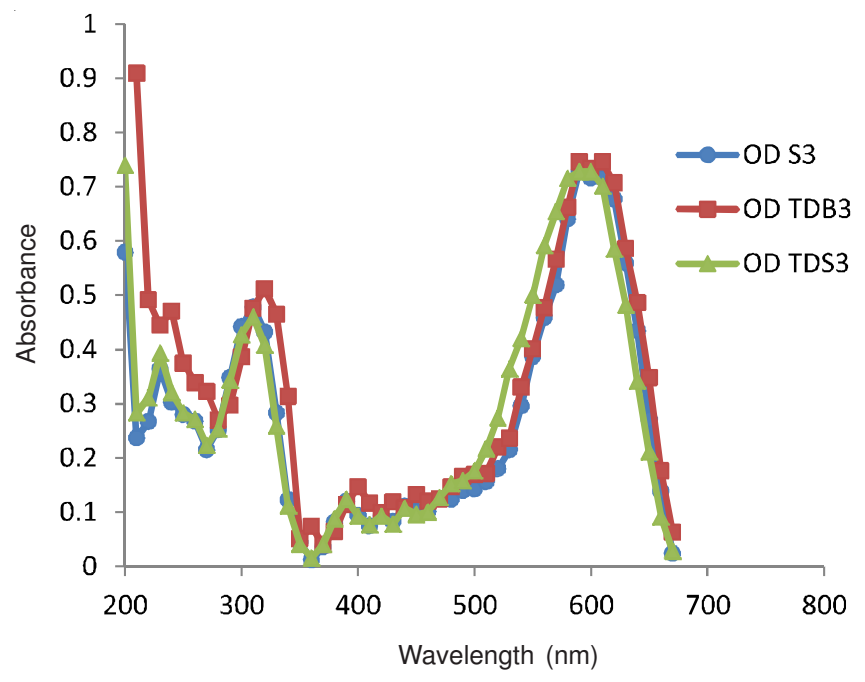

Fig. 3. Nanoparticles in dark changing absorbance of Reactive Red 190

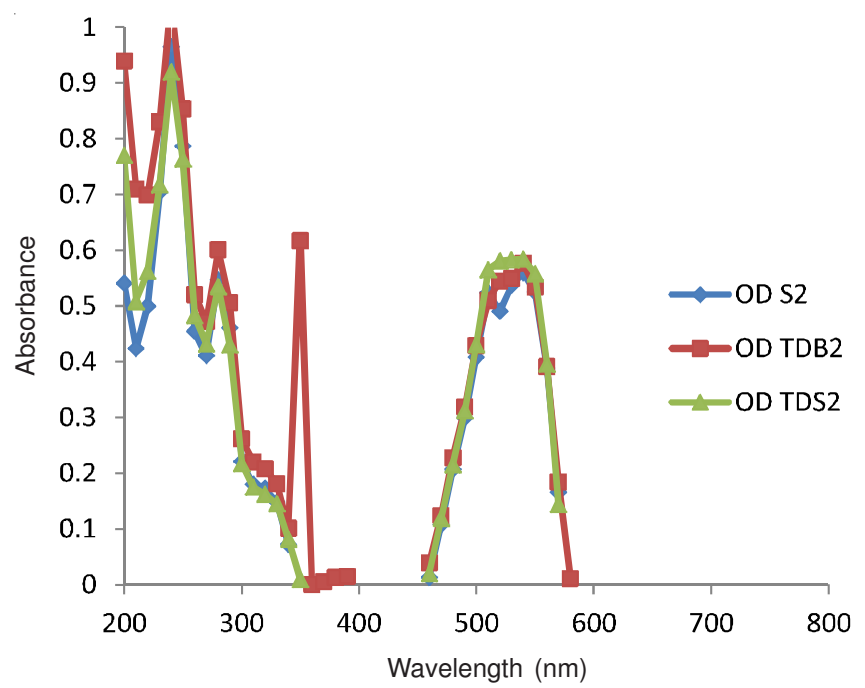

Fig. 4. Nanoparticles in dark changing absorbance of Reactive Black 5

Fig. 5 depicts the absorbance of Reactive Yellow 142 where OD S1 shows the absorbance of original dye without any treatment OD TDB1 shows the absorbance of dye treated in dark without nanoparticles and OD TDS1 shows the absorbance of dye treated in dark in the presence of nanoparticles.

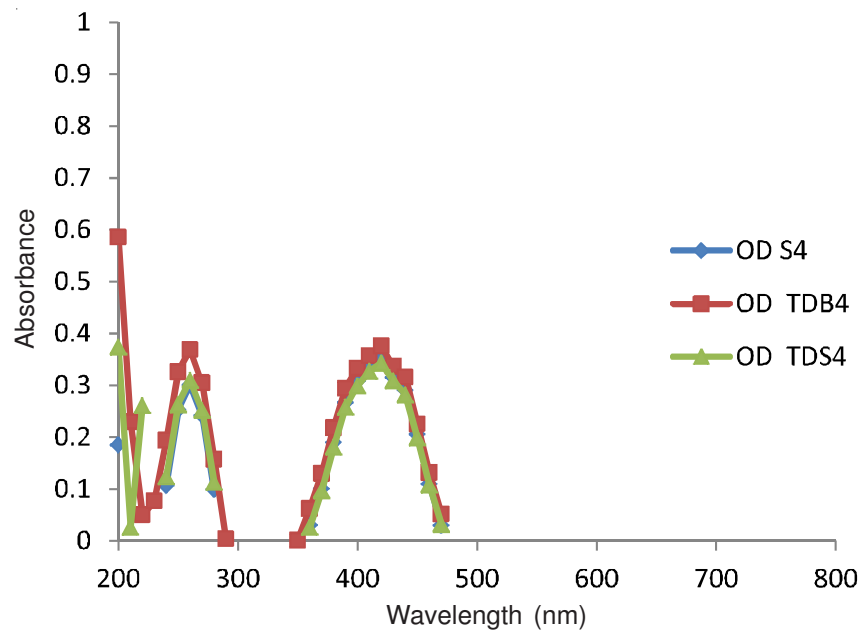

Fig. 5. Nanoparticles in dark changing absorbance of Reactive Yellow 142 
The effect of nanoparticles on dye revealed that absorbance of dye decreased when put in dark for degradation. The absorbance decreased on same trend. The absorbance of dye in visible region shifted from 0.4 to 0.3 hence the colour of treated dye was faded and there was a new peak between 200$250 \mathrm{~nm}$ which shows the change in chromophore. It took a slight blue shift.

Fig. 6 depicts the absorbance of Reactive Orange 13 in sunlight without nanoparticles at 30, 60, 90, 120 and $150 \mathrm{~min}$. The effect of time on dye solution in sunlight reveals that at zero minute the absorbance was less and it increased with time. This refers to the evaporation of water from the solution hence the solution became concentrated. Reactive Orange 13 shows absorbance in 200-600 $\mathrm{nm}$ where there is zero absorbance at places between 300 and 400. The increase in absorbance followed and continuous path.

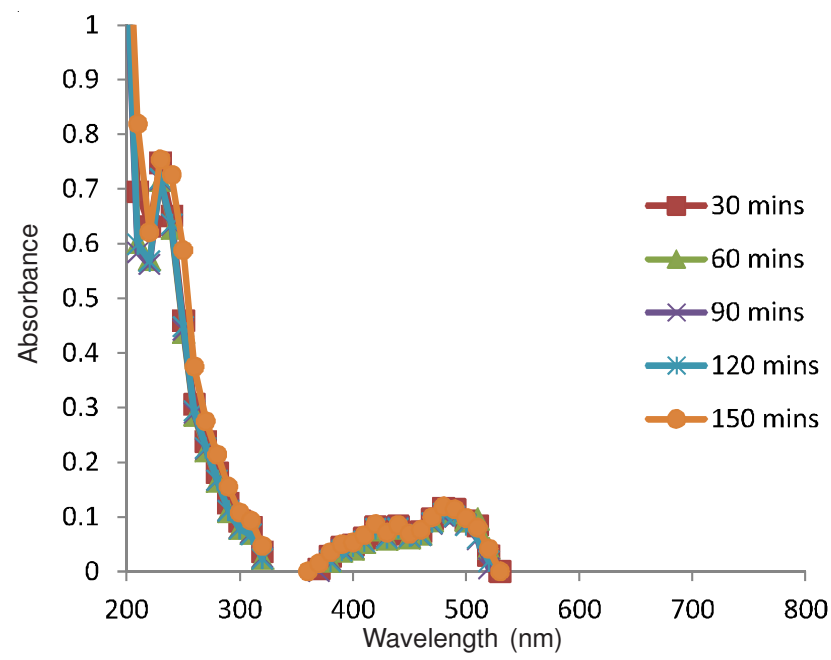

Fig. 6. Effect of time on Reactive Orange 13 in sunlight

Fig. 7 depicts the absorbance of Reactive Orange 13 in sunlight with nanoparticles at 30, 60, 90, 120, 150 min of treatment. The effect of time on dye colour when nanoparticles were added in sunlight revealed that absorbance of dye decreases with time. The dye shows absorbance in 200-400 nm. With passing time its absorbance decreases and decolourization occurred when $0.05 \mathrm{~g}$ nanoparticles were added. After $150 \mathrm{~min}$ the solution completely decolourized. There was no change in colour of nanoparticles which shows no apparent adsorption.

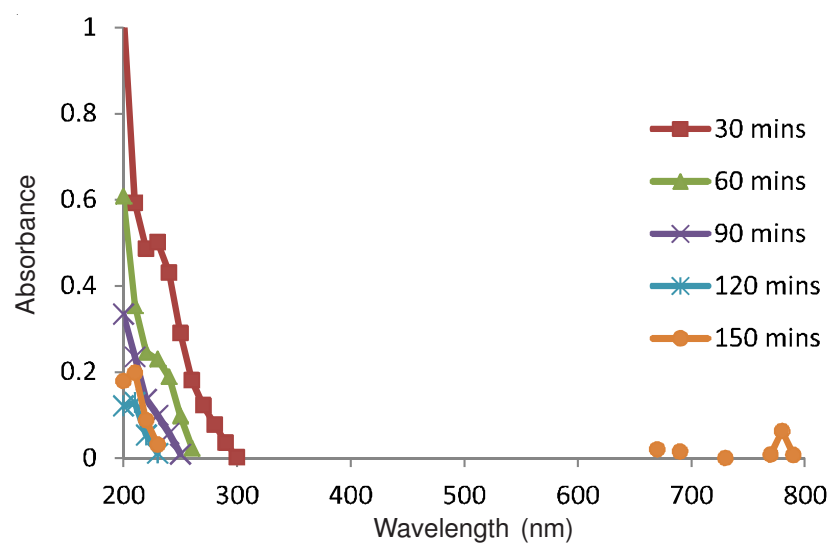

Fig. 7. Effect of nanoparticles with time in sunlight on Reactive Orange 13
Fig. 8 depicts the absorbance of Reactive Red 190 in sunlight without nanoparticles at 30, 60, 90, 120, $150 \mathrm{~min}$. The effect of time on dye solution in sunlight reveals that at zero minute the absorbance was less and it increased with time. This refers to the evaporation of water from the solution hence the solution became concentrated. The effect of time on dye solution in sunlight reveals that at zero minute the absorbance was less and it increased with time $150 \mathrm{~min}$. The dye shows absorbance in 200-600 $\mathrm{nm}$.

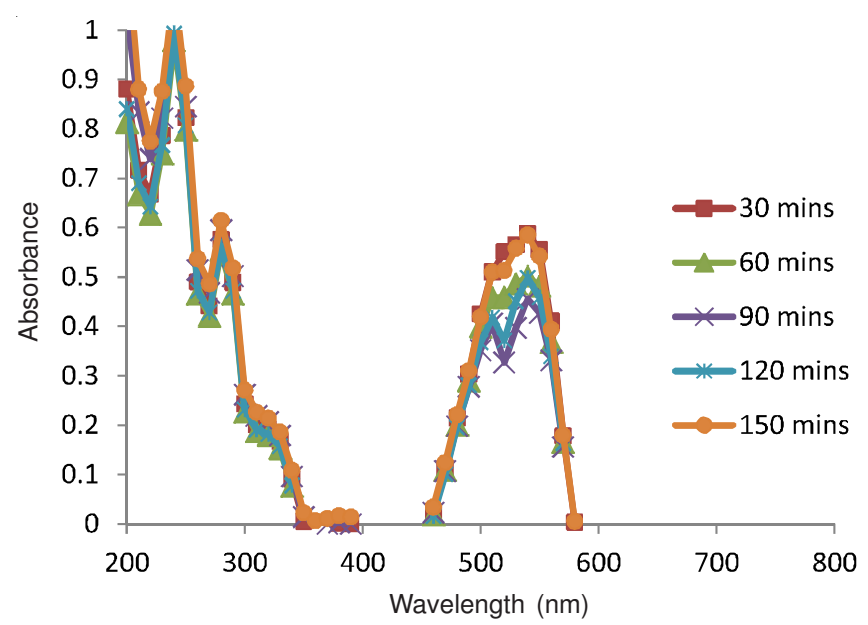

Fig. 8. Effect of time on Reactive Red 190 in sunlight

Fig. 9 depicts the absorbance of Reactive Red 190 in sunlight with nanoparticles at 30, 60, 90, 120, 150 min of treatment. The effect of time on dye colour when nanoparticles were added in sunlight revealed that absorbance of dye decreases with time. The dye shows absorbance in 200-600 $\mathrm{nm}$. With passing time its absorbance decreased and decolorization occurred when nanoparticles were added. After $3 \mathrm{~h}$ the solution completely decolourized. Peaks and absorbance trend remains the same which decreases on the same pattern as shown by the figure, the absorbance decreases as time increases. There was no absorbance in visible region after $0.5 \mathrm{~h}$ as the solution almost decolourized.

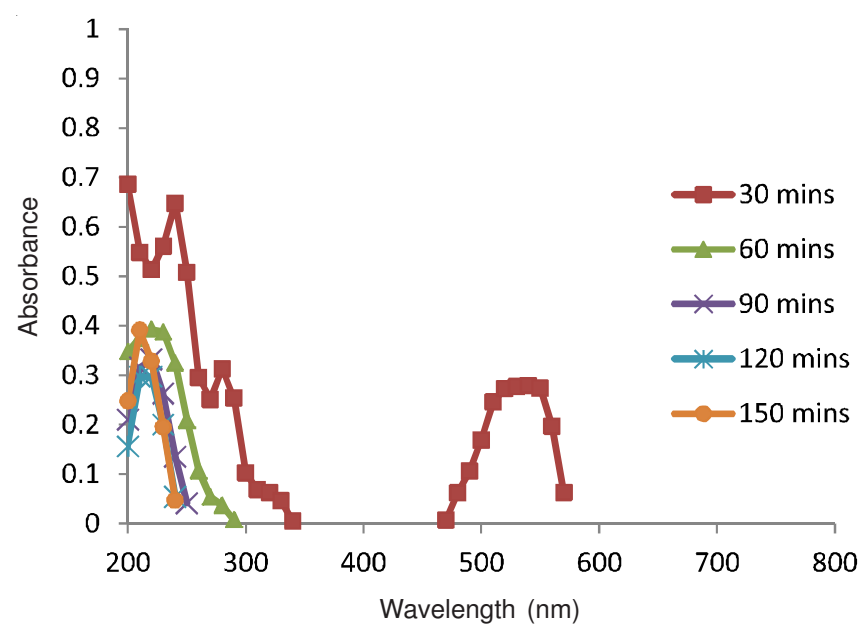

Fig. 9. Effect of nanoparticles with time in sunlight on Reactive Red 190

Fig. 10 depicts the absorbance of Reactive Black 5 in sunlight without nanoparticles at 30,60, 90, 120, $150 \mathrm{~min}$. 
The effect of time on dye solution in sunlight reveals that at zero minute the absorbance was less and it increased with time. This refers to the evaporation of water from the solution hence the solution became concentrated. The effect of time on dye solution in sunlight reveals that at zero minute the absorbance was less and it increased with time. The dye shows absorbance in 200-700 $\mathrm{nm}$. With passing time its absorbance increased on continuous path.

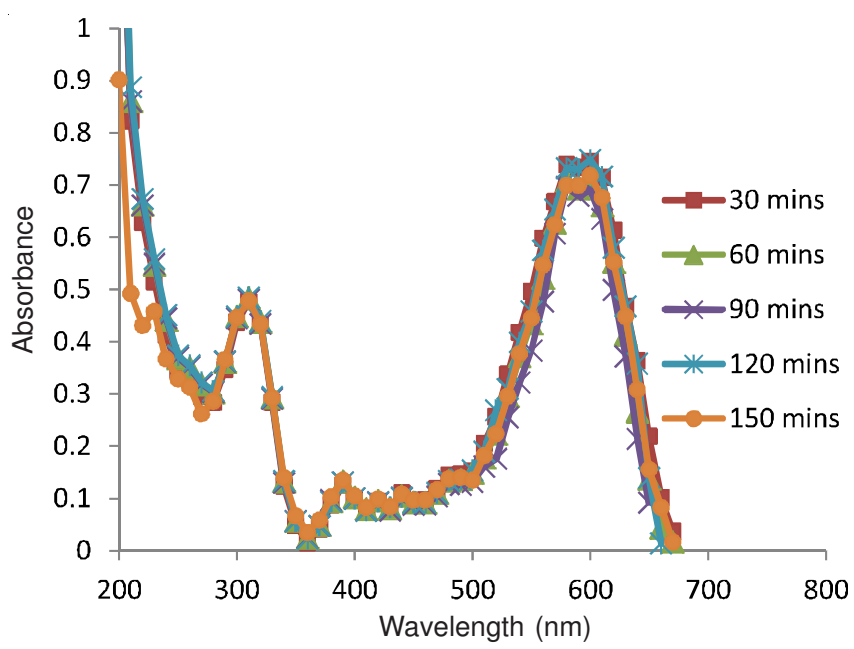

Fig. 10. Effect of time on Reactive Black 5 in sunlight

Fig. 11 depicts the absorbance of Reactive Black 5 in sunlight with nanoparticles at 30, 60, 90, 120, 150 min of treatment. The effect of time on dye colour when nanoparticles were added in sunlight revealed that absorbance of dye decreases with time. The dye shows absorbance in 200-700 $\mathrm{nm}$. With passing time its absorbance decreased and decolorization occurred when nanoparticles were added. After $3 \mathrm{~h}$ the solution completely decolourized. The absorbance decreases as time increases. There was no absorbance after $90 \mathrm{~min}$ in visible region yet at $150 \mathrm{~min}$ there were some points in 700$800 \mathrm{~nm}$ due to appearance of some chromophore. The dye completely decolourized after $150 \mathrm{~min}$.

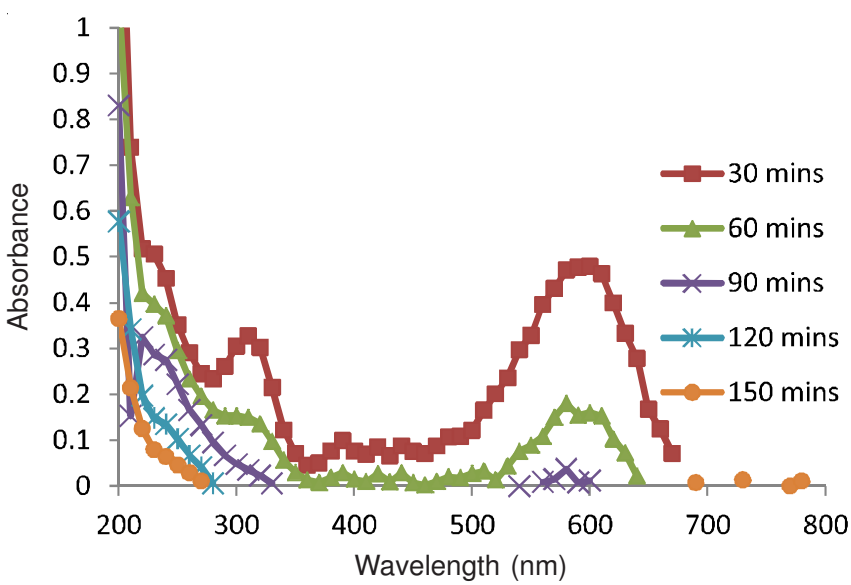

Fig. 11. Effect of nanoparticles with time in sunlight on Reactive Black 5

Fig. 12 depicts the absorbance of Reactive Yellow 142 in sunlight without nanoparticles at 30, 60, 90, 120, $150 \mathrm{~min}$. The effect of time on dye solution in sunlight reveals that at zero minute the absorbance was less and it increased with time.
This refers to the evaporation of water from the solution hence the solution became concentrated. The effect of time on dye solution in sunlight reveals that at zero minute the absorbance was less and it increased with time $150 \mathrm{~min}$. The dye shows absorbance in 200-500 $\mathrm{nm}$. With passing time its absorbance increased on continuous path. There was a new peak after $1 \mathrm{~h}$ near $500 \mathrm{~nm}$ which shows some excited group.

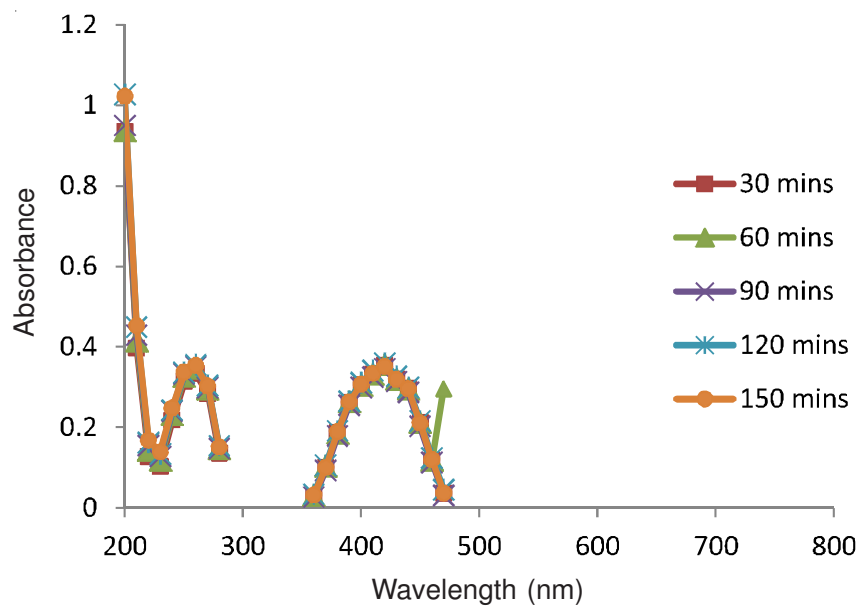

Fig. 12. Effect of time on Reactive Yellow 142 in sunlight

Fig. 13 depicts the absorbance of Reactive Yellow 142 in sunlight with nanoparticles at 30, 60, 90, 120, 150 min of treatment. The effect of time on dye colour when nanoparticles were added in sunlight revealed that absorbance of dye decreases with time. The dye shows absorbance in 200-500 $\mathrm{nm}$. With passing time its absorbance decreased and decolorization occurred when nanoparticles were added. After $3 \mathrm{~h}$ the solution completely decolourized. The absorbance decreases as time increases. The dye completely decolourized after 150 min. There was no apparent change in colour of nanoparticles which shows that no adsorption occurred.

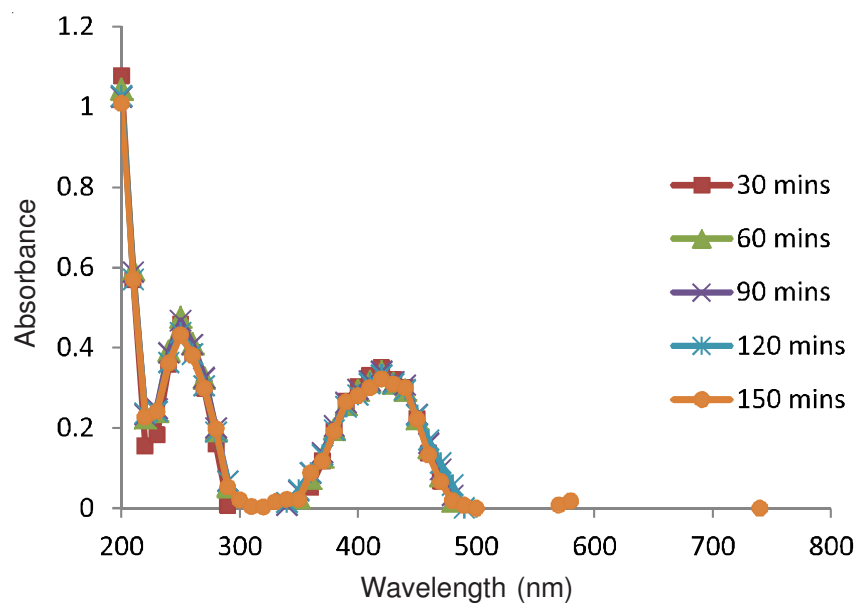

Fig. 13. Effect of nanoparticles with time in sunlight on Reactive Yellow 142

Fig. 14 depicts the absorbance of Reactive Orange 13 in sunlight without nanoparticles at 30, 60, 90, 120, $150 \mathrm{~min}$. The effect of time on dye solution in UV light reveals that at zero minute the absorbance was less and it increased with time. The dye shows absorbance in 200-600 $\mathrm{nm}$. There was an 


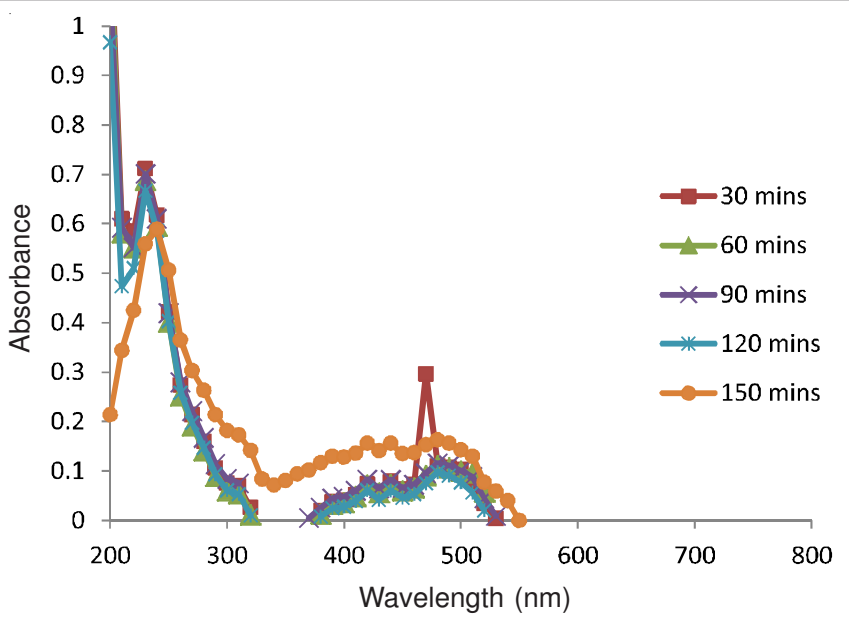

Fig. 14. Effect on the absorbance of Reactive Orange 13 in UV

unusual peak near $500 \mathrm{~nm}$ at $0.5 \mathrm{~h}$ which became extinct afterwards. There was a slight red shift in absorbance of dye and a continuous absorbance from 200 to near $600 \mathrm{~nm}$ appeared after $150 \mathrm{~min}$.

Fig. 15 depicts the absorbance of Reactive Orange 13 in UV with nanoparticles at 30,60, 90, 120, 150 min of treatment. The effect of time on dye colour when nanoparticles were added in UV revealed that absorbance of dye decreases with time. The dye shows absorbance in 200-600 nm. With passing time its absorbance decreased and decolorization occurred when nanoparticles were added. After $3 \mathrm{~h}$ the solution completely decolourized. The absorbance decreases as time increases. There was a new peak formed at $2 \mathrm{~h}$ due to excitation. There was no apparent change in colour of nanoparticles which shows that no adsorption occurred.

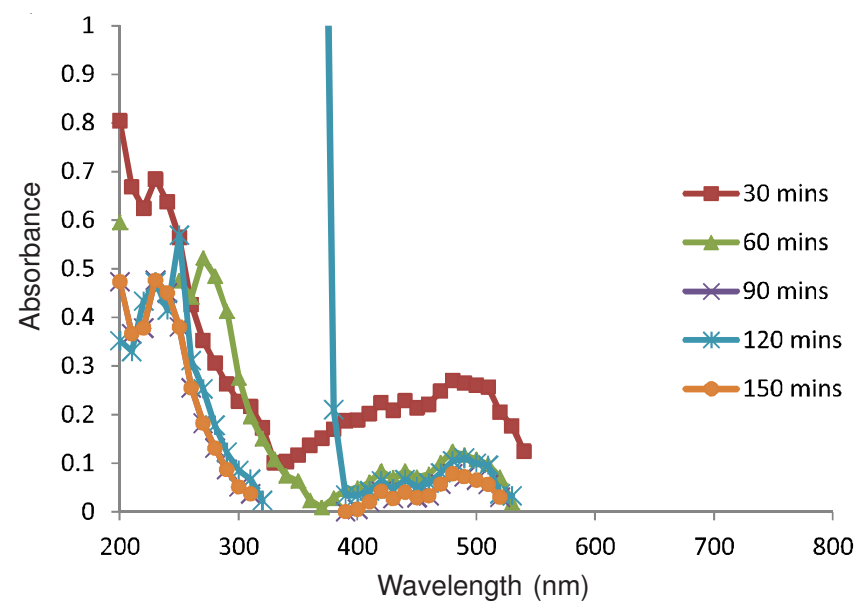

Fig. 15. Effect of nanoparticles in change in absorbance of Reactive Orange 13 in UV

Fig. 16 depicts the absorbance of Reactive Red 190 in UV without nanoparticles at 30,60, 90, 120, $150 \mathrm{~min}$. The effect of time on dye solution in UV light reveals that at zero minute the absorbance was less and it increased with time. The dye shows absorbance in 200-600 $\mathrm{nm}$. There was an unusual peak near $200 \mathrm{~nm}$ at $150 \mathrm{~min}$ which became extinct afterwards. There was a slight red shift in absorbance of dye and a continuous absorbance from 200 to near $600 \mathrm{~nm}$ appeared after $150 \mathrm{~min}$.

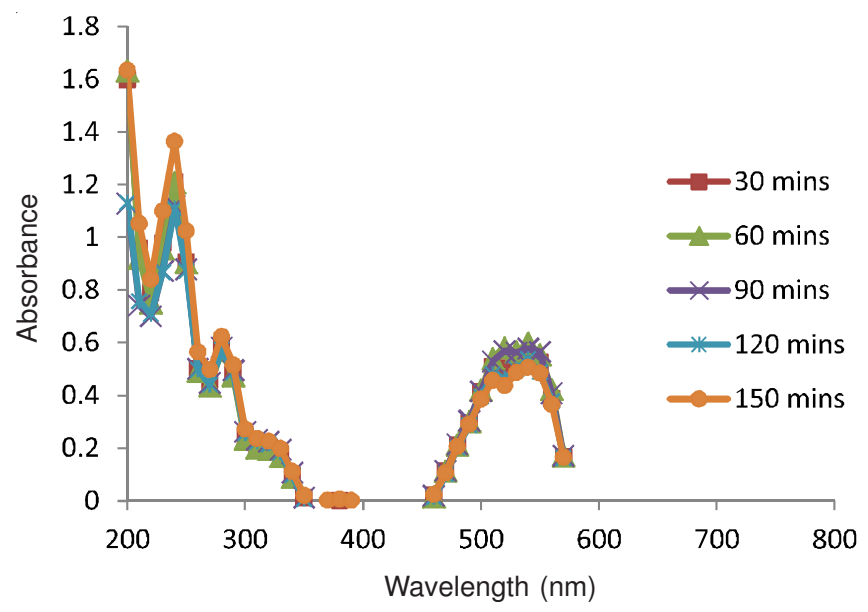

Fig. 16. Effect on absorbance of Reactive Red 190 in UV

The effect of time on dye solution in UV light reveals that there was change in absorbance of dye when nanoparticles were added in it. There was a break in continuous spectrum of absorbance which shows a change in chromophore structure.

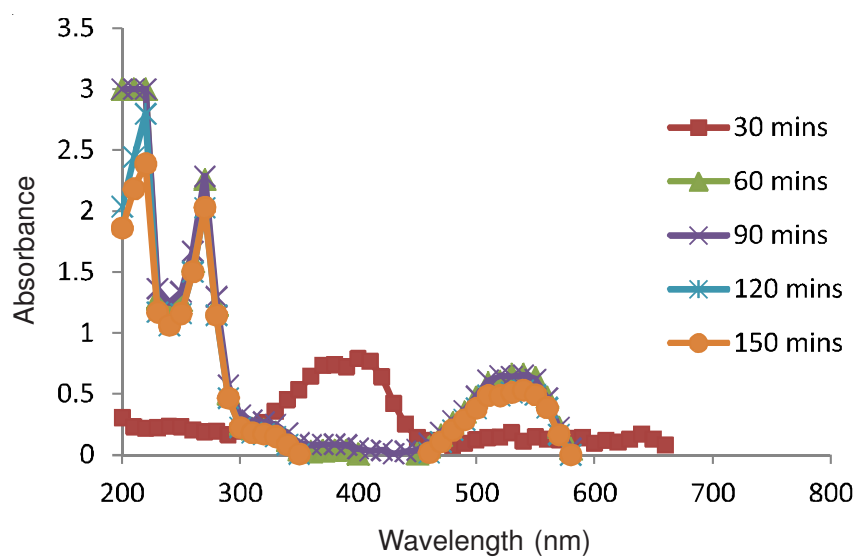

Fig. 17. Effect of nanoparticles in change in absorbance of Reactive Red 190 in UV

Fig. 18 depicts the absorbance of Reactive Black 5 in UV without nanoparticles at 30,60, 90, 120, $150 \mathrm{~min}$. The effect of time on dye solution in UV light reveals that at zero minute the absorbance was less and it increased with time. The dye shows absorbance in $200-700 \mathrm{~nm}$.

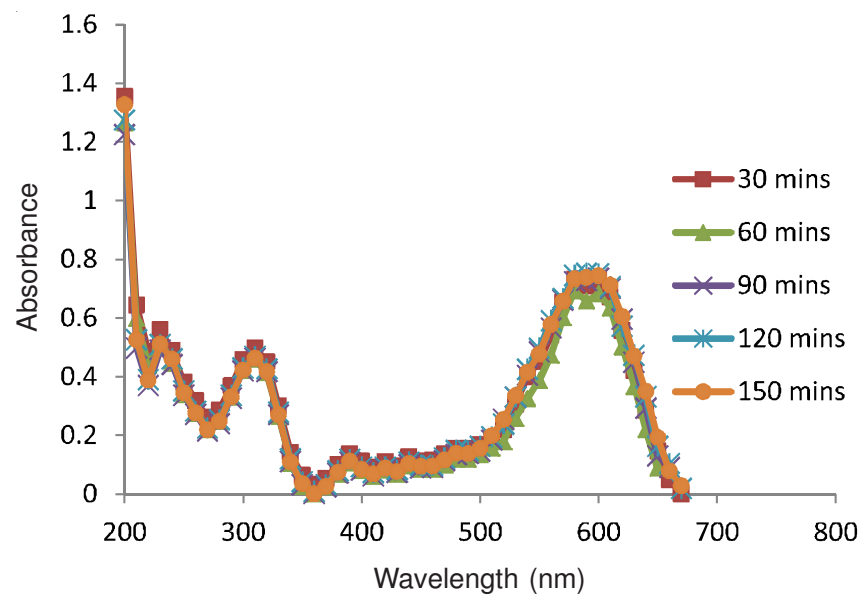

Fig. 18. Effect on the absorbance of Reactive Black 5 in UV 
Fig. 19 depicts the absorbance of Reactive Black 5 in UV with nanoparticles at 30,60, 90, 120, 150 min of treatment. The effect of time on dye colour when nanoparticles were added in UV revealed that absorbance of dye decreases with time. The dye shows absorbance in 200-600 nm. With passing time its absorbance decreased and decolourization occurred when nanoparticles were added. After $3 \mathrm{~h}$ the solution completely decolorized. The absorbance decreases as time increases. There was a new peak formed at $1 \mathrm{~h}$ due to excitation which became extinct afterwards. There was no apparent change in colour of nanoparticles which shows that no adsorption occurred.

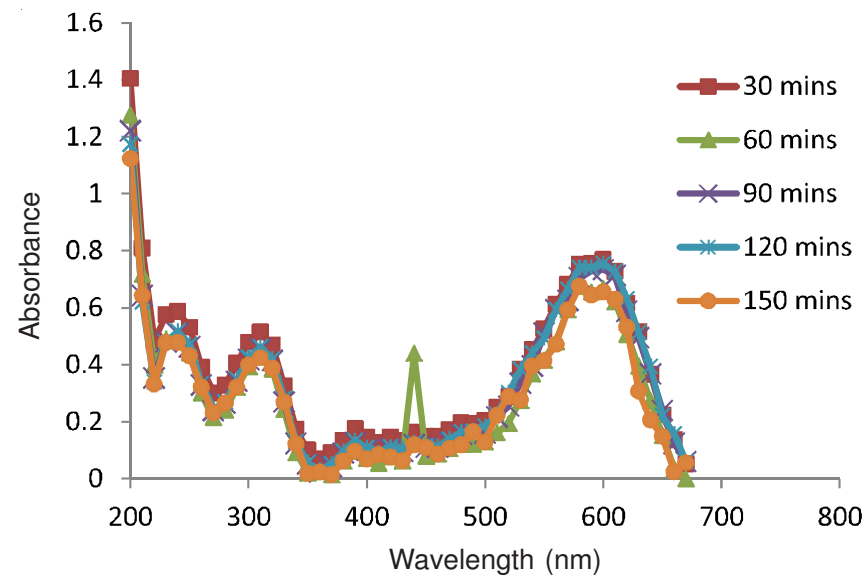

Fig. 19. Effect of nanoparticles in change in absorbance of Reactive Black 5 in UV

Fig. 20 depicts the absorbance of Reactive Yellow 142 in UV without nanoparticles at 30, 60, 90, 120, $150 \mathrm{~min}$. The effect of time on dye solution in UV light reveals that at zero minute the absorbance was less and it increased with time. The dye shows absorbance in 200-700 nm.

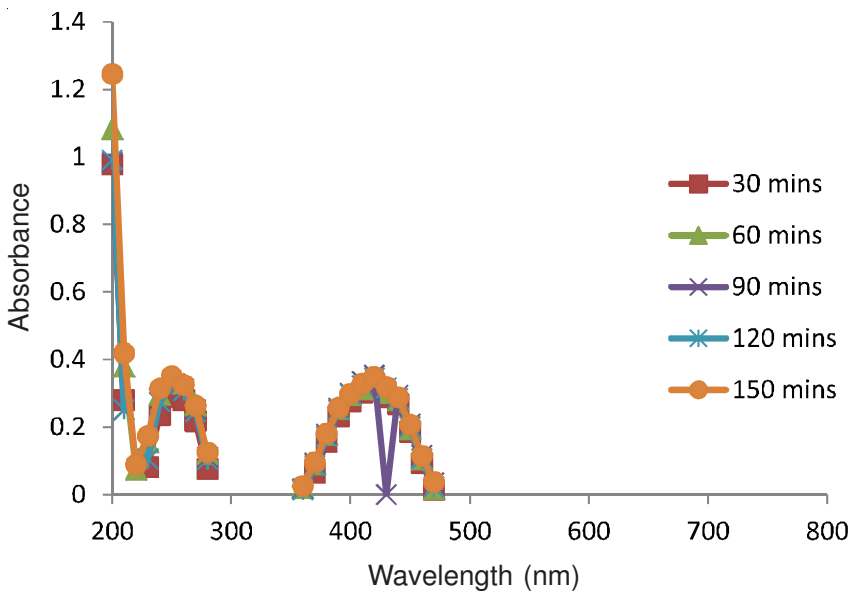

Fig. 20. Effect on the absorbance of reactive tellow 142 in UV

Fig. 21 depicts the absorbance of Reactive Yellow 142 in UV with nanoparticles at 30, 60, 90, 120, 150 min of treatment. The effect of time on dye colour when nanoparticles were added in UV revealed that absorbance of dye decreases with time. The dye shows absorbance in 200-500 $\mathrm{nm}$. With passing time its absorbance decreased and decolourization occurred when nanoparticles were added. After $3 \mathrm{~h}$ the solution completely decolourized. The absorbance decreases as time increases.

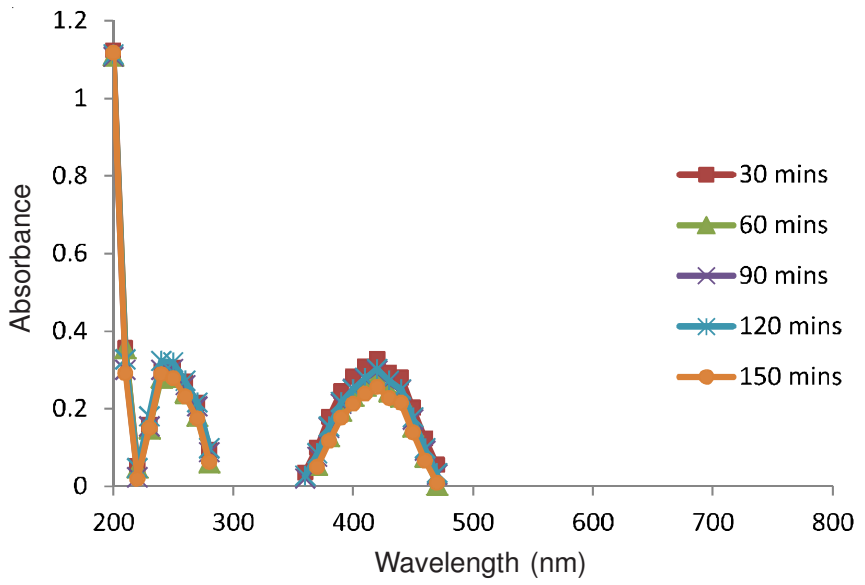

Fig. 21. Effect of nanoparticles in change in absorbance of Reactive Yellow 142 in UV

There was no apparent change in colour of nanoparticles which shows that no adsorption occurred. Fig. 22 shows the pH of Reactive Orange 13, Reactive Red 190, Reactive Black 5 and Reactive Yellow 142. The error bars show the fluctuations in $\mathrm{pH}$ of all four dyes. The trend shows that $\mathrm{pH}$ of untreated samples was neutral but after the addition of nanoparticles it decreased and became acidic.

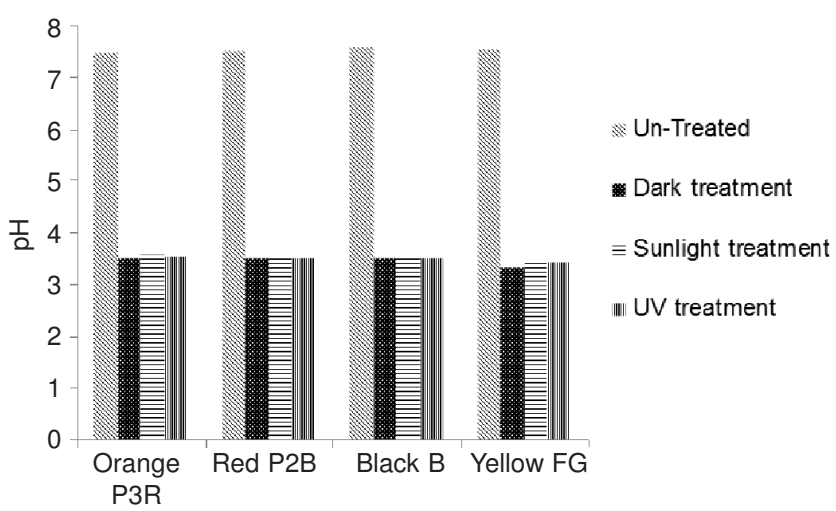

Fig. 22. Effect of $\mathrm{pH}$

Table- 2 shows the per cent removal of TOC which decreased in all three treatments.

Reactive dye are most extensively used group of azo dyes as they give good colour and have a broad colour range. Moreover they are easy to use, relatively low cost and require less energy. By products of these dyes: aromatic amines are harmful for aquatic life they can even cause cancer in humans. So their treatment is necessary in wastewater.

Table- 1 shows the per cent removal of COD in all three treatments. COD decreased in all three treatments.

In this study synthesis of titanium dioxide nanoparticles has done through coprecipitation method. Comparatively less work is cited in this field previously as the un-doped nanoparticles in powder form are used in this research which decolourize the dyes solutions. These nanoparticles were then characterized through $\mathrm{X}$ ray diffraction technique. XRD analysis was performed using Braco diffractometer at radiation of $\mathrm{CuK}_{\alpha}$ in a $\theta-2 \theta$ configuration for already prepared titanium dioxide nanoparticles to study their size, structure and composition. 
TABLE-1

REMOVAL \% OF COD OF TREATED SAMPLES

\begin{tabular}{lc}
\hline \multicolumn{1}{c}{ Sample } & Dark treatment $(\%$ removal $)$ \\
\hline Reactive orange 13 & 13 \\
Reactive red 190 & 19 \\
Reactive black 5 & 14 \\
Reactive yellow 142 & 13 \\
\hline
\end{tabular}

TABLE-2

REMOVAL \% OF TOC OF TREATED SAMPLES

\begin{tabular}{lc}
\hline \multicolumn{1}{c}{ Sample } & Dark treatment (removal \%) \\
\hline Reactive orange 13 & 11 \\
Reactive red 190 & 15 \\
Reactive black 5 & 13 \\
Reactive yellow 142 & 10 \\
\hline
\end{tabular}

Obtained intensity readings of titania nanoparticle sample are plotted against angle of diffraction (Fig. 1). The narrow and fine peaks indicate that nanoparticles are well crystallized. There are no significant peaks showing impurities. Peak analysis showed that titania nanoparticles were of anatase phase. According to Debye Scherrer's formula the size of nanoparticles was calculated which shows that average size of nanoparticles was between $50-70 \mathrm{~nm}$.

The prepared nanoparticles were used for the treatment of synthetic dye effluent. Four dyes viz., Reactive Orange 13, Reactive Red 190, Reactive Black 5 and Reactive Yellow 142 were used. These dyes were treated in dark.

Figs. 2-5 show the effect of nanoparticles in dark. It is significant from UV visible spectroscopy that when nanoparticles were added in them the absorbance of all four dyes decreased due to degradation and there COD and TOC also decreased. The colour of nanoparticles was not changed which shows that no adsorption occurred. The $\mathrm{pH}$ of the dyes was measured before and after treatment which was 7.45, 7.48, 7.55, 7.53 of Reactive Orange 13, Reactive Red 190, Reactive Black 5, Reactive Yellow 142, respectively which became after treatment 3.50, 3.49, 3.48, 3.33 (Fig. 22). It is observed that $\mathrm{pH}$ became acidic when nanoparticles of titania were added in effluent, this is due to acidic nature of titania nanoparticles.

Figs. 6-13 show the effect of sunlight on dyes effluents. When no nanoparticles were added in the solution the colour was not removed and their absorbance increased. This can be due to evaporation of water from the solution thus concentrating the solution. When nanoparticles were added in the effluents the absorbance decreased and dye were decolourized. The effect of nanoparticles was studied as a function of time. The degradation rate was found to increase with the increase in irradiation time. The $100 \%$ degradation was achieved in 150 min however this time is different for different dyes as some dyes decolourized in first 30 or 90 min which resulted in overlapping of graph lines as shown in above figures still all dyes were given $150 \mathrm{~min}$ treatment to make the procedure effect constant. The $\mathrm{pH}$ of the solution was observed before and after treatment which was 7.45, 7.48, 7.55 and 7.53 of Reactive Orange 13, Reactive Red 190, Reactive Black 5 and Reactive Yellow 142, respectively which became after treatment 3.55, $3.51,3.49,3.43$, respectively. It was observed that $\mathrm{pH}$ was neutral before treatment and became acidic after addition of nanoparticles. When the dyes were directly exposed to sunlight without addition of titania nanoparticles no decolourization was observed (Figs. 6, 8, 10, 11 and 13). It is also observed that COD decreases slower than decolourization of the solution (Table-1). This is due to the fact that dyes are not directly mineralized but transform in to intermediate photoproducts. The COD removal of all four dye Reactive Orange 13, Reactive Red 190, Reactive Black 5 and Reactive Yellow 142 in sunlight treatment was 29,20, 25 and $24 \%$, respectively. Titania nanoparticles are photosensitive and are effective in degrading selected azo dyes completely in a short interval of time (150 $\mathrm{min})$. The cleavage of $\mathrm{C}=\mathrm{C}$ double bonds and other functional groups will shift the absorption spectra of the molecule out of the visible region. The TOC of all four dyes Reactive Orange 13, Reactive Red 190, Reactive Black 5 and Reactive Yellow 142 decreased with a percent of 18,20, 20 and $19 \%$, respectively. Peaks and absorbance though decreased but their trend remained as seen in figures. The absorbance decreases as time increases. There was no change in apparent colour of nanoparticles which shows that no adsorption occurred.

Figs. 14-21 show the effect of UV light on dyes effluents. It was observed that in the absence of nanoparticles the absorbance of dye increased (Figs. 14, 16, 18 and 20). But when nanoparticles were added the absorbance decreased (Figs. 15, 17, 19 and 21). It is because organic molecules absorb UV energy are turn into the excited state thus become more susceptible to attack. Therefore, the rate at which organic compounds are oxidized is significantly higher than that attained by using UV irradiation alone so the decolourization occurs maximum in UV when nanoparticles are added to the solution. UV efficiency appears to be a function of irradiation time. In the case of effluent COD removal is evident at UV irradiation time of $150 \mathrm{~min}$ and TOC was also decreased. The COD removal of all four dyes Reactive Orange 13, Reactive Red 190, Reactive Black 5 and Reactive Yellow 142 in UV treatment was $15,17,14$ and $12 \%$, respectively. The TOC of all four dyes Reactive Orange 13, Reactive Red 190, Reactive Black 5 and Reactive Yellow 142 decreased with a per cent of 13, 12, 11 and $10 \%$, respectively.

Results of CHOMPUCHAN (2009) also depict the decolourization of azo dyes with iron nanoparticles. With all three processes we found effective decolourization of dyes. It also found $\mathrm{TiO}_{2}$ capable to decolourize wastewater ${ }^{13}$.

\section{Conclusion}

The results of the present investigation elucidate the potential health hazard associated with these dyes. It was found that all four dyes can be treated efficiently with titania nanoparticles. This protocol is an effective technique in decolorizing these azo dye solutions and can be employed effectively in the treatment of textile dye effluents which are hazardous to the environment. The effect of nanoparticles on different dyes with the function of time was observed. The time effects positively on colour removal of dyes with increase in time the colour removal increases.

\section{ACKNOWLEDGEMENTS}

The authors are thankful to Dr. Haq Nawaz Bhatti Department of Chemistry and Biochemistry University of 
Agriculture Faisalabad for their kind cooperation. The authors are also thankful to staff of PIEAS Nilore Islamabad, Pakistan for providing facility to use their laboratories.

\section{REFERENCES}

1. A.S. Malik, Impact of Environmental Regulations on the Textile Sector of Pakistan. Conference Held October 2-4, 2002, Geneva, Switzerland (2002).

2. C.D. Rail, Groundwater Contamination: Sources, Control and Preventive Measures, Technomic Publishing Co., Lancaster, PA, USA, edn. 1, pp. 1-139 (1989).

3. I. Arslan, I.A. Balcioglu and D.W. Bahnemann, Appl. Catal. B: Environ., 26, 193 (2000)

4. T. Sauer, G. Cesconeto Neto, H.J. Jose and R.F.P.M. Moreira, J. Photochem. Photobiol. A: Chem., 149, 147 (2002).
5. H. Zollinger, Synthesis, Properties and Applications of Organic Dyes and Pigments, VCH Publishers, New York, USA, pp. 92-100 (2002).

6. P.S. Melo, J.B. Fabrin-Neto, S.G. De Moraes, M.R. Assalin, N. Duran and M. Haun, Chemosphere, 62, 1207 (2006).

7. A. Al-Kdasi, A. Idris, K. Saed and C.T. Guan, Global Nest: Int. J., 6, 222 (2004).

8. U. Pagga and D. Brown, Chemosphere, 15, 479 (1986).

9. T.H. Bui, M. Karkmaz, E. Puzenat, C. Guillard and J.M. Herrmann, Res. Chem. Intermed., 33, 421 (2007).

10. C. Zhu, L. Wang, L. Kong, X. Yang, S. Zheng, F. Chen, F. MaiZhi and H. Zong, Chemosphere, 41, 303 (2000).

11. C.H. Wu and J.M. Chern, Ind. Eng. Chem. Res., 45, 6450 (2006).

12. L.S. Clesceri, A.E. Greenberg and A.D. Eaton, Standard Methods for the Examination of Water and Wastewater, American Public Health Association, Washington, USA, edn. 20, p. 1325 (1998).

13. F. Han, V.S.R. Kambala, M. Srinivasan, D. Rajarathnam and R. Naidu, Appl. Catal. A: Gen., 359, 25 (2009). 